\title{
Optimum Structure of multi-layer silver superlenses for optical lithography
}

\author{
Junxian Ma \\ Advanced Technology Research Centre \\ Shenzhen University \\ Shenzhen, China \\ majx@szu.edu.cn
}

\author{
Kuangying Yuan \\ Advanced Technology Research Centre \\ Shenzhen University \\ Shenzhen, China \\ yuanjing19871203@163.com
}

\begin{abstract}
Multilayer silver superlenses had been shown to having sub-wavelength performance. In this paper, we investigate the effects on imaging performance of different multilayer imaging structures comprising alternately layered metal and dielectric films. We first compare and analyze transmission coefficients of different structures by changing the layer thickness ratio, the total thickness of the layers and the dielectric materials. Then we simulate the imaging performance by the finite difference time domain (FDTD) method of these multilayered superlenses. According to the analyzing and simulated results, a multilayer silver superlens with optimum structure is suggested.
\end{abstract}

Keywords-Superlens; Surface plasmons; Photolithography; Imaging performance

\section{INTRODUCTION}

Superlens was first proposed by Pendry in 2000, which can be obtained by a thin slab of negative refraction index material [1]. A thin silver slab was his realistic suggestion for a practical near-field superlens. It works by enhancing and coupling surface plasmon polaritons (SPPs) across thin layers of silver, interleaved with layers of dielectric. Since then, many works on this superlens have been done by reseachers from all the world. Based on Pendry's work, S.A.Ramakreshna proposed a multilayer stack of positiveand negative- refractive-index media, in which, the normally evanescent parts are now transmitted, and there is a preferred direction of propagation [2]. Simulation results exploring the use of superlenses in near field optical nanolithography are presented by R.J. Blaikie, which opens up the possibility of proximity near-field optical nanolithography [3]. Then these concept were experimentally verified by David O.S. Melville using a novel near-field lithographic technique called Planar Lensing Lithography (PLL), and resolution down to $40 \mathrm{~nm}$ has been predicted [4].

For these and other studies there is a need to analyze the imaging performance of the superlenses, to determine the best lens structures for subdiffraction-limited imaging and to characterize experimental performance degradation due to system imperfections. To date, the analysis has largely been concentred on the layer thickness and layer numbers [5-10]. However, some other aspects of the superlenses' structures such as the thickness ratio of adjacent layer and the dielectric materials should be taken into account.

In this paper, we present an analysis of the imaging performance of various multilayer silver-dielectric superlenses. The transfer functions, which show the magnitude of light transmitted by the lens over a range of spatial frequencies, are used to evaluate the imaging performance of the superlenses. A finite difference time domain (FDTD) method is used for simulation of the nanolithography. Then we present the results of our analysis, and compare the performance of superlenses with different total layer thickness, different dielectric material and different thickness ratio of two adjacent layer. At last, we suggest a optimum structures according to the analysis results.

\section{THEORY AND STRUCTURES OF MULTILAYERED SUPERLENS}

A near-field optical photolithographic scheme based on multilayered metal-dielectric superlens is shown in Fig. 1. Here, a $60 \mathrm{~nm}$ thick grating chromium mask is patterned on a conformable glass mask holder and incorporating multilayer silver superlens stack. Then a $350 \mathrm{~nm}$ thick positive photoresist is spun onto the silicon substrate to record the near field image of the grating mask. For the superlens stack, $\varepsilon_{m}$ and $\varepsilon_{d}$ are the permittivity of the dielectric and metal, respectively, while $d_{1}$ and $d_{2}$ are the thickness of the corresponding layers, $\varepsilon$ is the permittivity of the surrounding medium. The wavelength of the incident plane wave is $\lambda$.

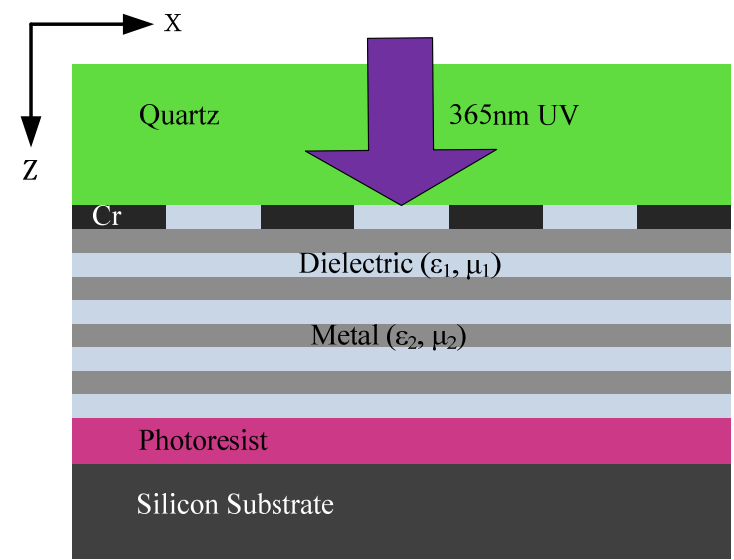

Figure 1. Schematic construction of a multilayer superlens that used in near field optical lithograghy imaging experiments.

We assume that each layer can be described by homogeneous and isotropic permittivity and permeability parameters. According to the effective medium theory (EMT), when the layers are sufficiently thin, the layered 
metal-dielectric superlens can be treated as a single anisotropic medium [6-8]. The transverse and longitudinal permittivity can be respectively described as

$$
\begin{gathered}
\varepsilon_{x}=\varepsilon_{y}=\frac{\varepsilon_{m}+\eta \varepsilon_{d}}{1+\eta} \\
\varepsilon_{z}=\frac{(1+\eta) \varepsilon_{m} \varepsilon_{d}}{\eta \varepsilon_{m}+\varepsilon_{d}}
\end{gathered}
$$

Where $\eta$ is the ratio of the adjacent two layer thickness

$$
\eta=\frac{d_{1}}{d_{2}}
$$

$d_{1}$ and $d_{2}$ are the thickness of the metal layer and dielectric layer respectively. The homogenized magnetic permeability is given by expressions analogous to (1) and (2). When $\eta$ is small, the effective parameters are dominated by the first medium, while for large $\eta$, they resemble those of the second medium. The slab material data from which these plots are constructed have been assumed to be Drude model, as given below

$$
\varepsilon(\omega)=\varepsilon_{0}-\frac{\omega_{p}^{2}}{\omega\left(\omega+i \gamma_{p}\right)}
$$

Where $\varepsilon_{0}$ is the contribution due to interband transitions, $\omega_{p}$ is the bulk plasma frequency and $\gamma_{p}$ is the relaxation constant. The imaging performance of the multilayered superlenses can be understood by calculating the transmission coefficient $t$ for a given input intensity at a given spatial frequency or wavenumber $\left(k_{x}\right)$. We assume that we are dealing with nonmagnetic materials, so that the magnetic permeability $\mu=1$. If the dielectric is anisotropic, the interesting waves are those with transverse magnetic (TM) polarization. The tansmission coefficient for these waves is

$$
t\left(k_{x}\right)=\frac{1}{\cos k_{z} d-\frac{i}{2}\left(\frac{k_{z} \varepsilon}{k_{z}^{\prime} \varepsilon_{x}}+\frac{k_{z}^{\prime} \varepsilon_{x}}{k_{z} \varepsilon}\right) \sin k_{z} d}
$$

Where $d$ is the total thickness of the multilayered superlens. $k_{z}=\sqrt{\left(k_{0}^{2}-k_{x}^{2} / \varepsilon_{z}\right) \varepsilon_{x}}$ is the wave vector of the superlens and $k_{z}^{\prime}=\sqrt{\varepsilon k_{0}{ }^{2}-k_{x}{ }^{2}}$ is the longitudinal wave vector of the surrounding.

\section{COMPARISON AND ANALYSIS}

In this section, we analyze the performance of various multilayer silver-dielectric lens stacks. We vary the structures of the superlenses by changing the total thickness of the layers, the thickness ratio of metal to dielectric and the materials of dielectric. Firstly, we consider the effect on imaging performance of varying the layer thickness ratios within a multilayer superlens. Then we change the total thickness of the superlenses to achieve a optimum imaging performance. At last, we present the imaging performance for different dielectric materials, while the metal material is Ag.

\section{A. Thickness Ratio of Dielectric to Metal}

For a layered metal-dielectric superlens, when the components of this permittivity have opposite signs, we can model this superlens as a homogeneous effective medium with anisotropic dielectric permittivity. According to the theory present in section II, we can tune the response either by altering the frequency or by changing the ratio of layer thicknesses. The permittivity of the silver ( $\mathrm{Ag}$ ) can be obtained by (4) with $\varepsilon_{0}=6.0, \omega_{p}=1.5 \times 10^{16} \mathrm{rad} / \mathrm{s}$, $V_{c}=7.73 \times 10^{13} \mathrm{rad} / \mathrm{s}$, while the silica has permittivity $\varepsilon_{d}=2.5$ at $365 \mathrm{~nm}$ working wavelength. The total thickness of the superlens is set to $60 \mathrm{~nm}$.

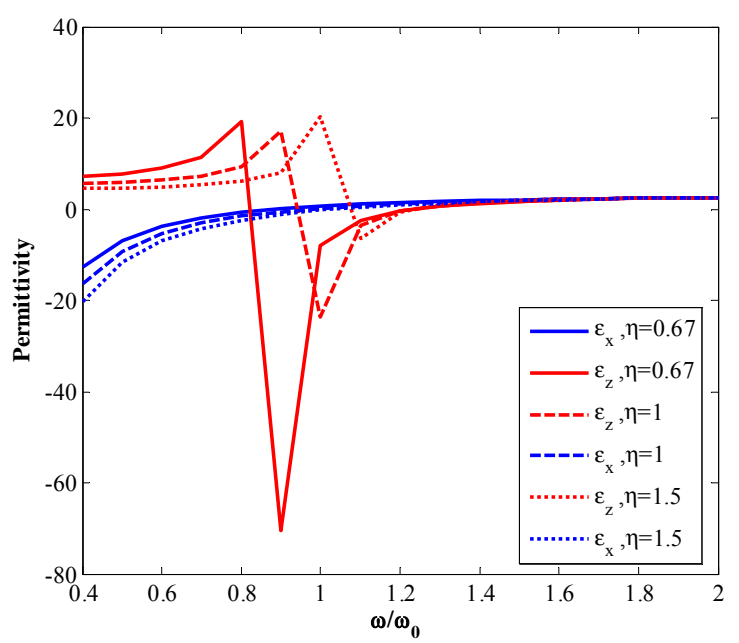

Figure 2. The dielectric permittivity of the metamaterial constructed from layers of silver and silica.

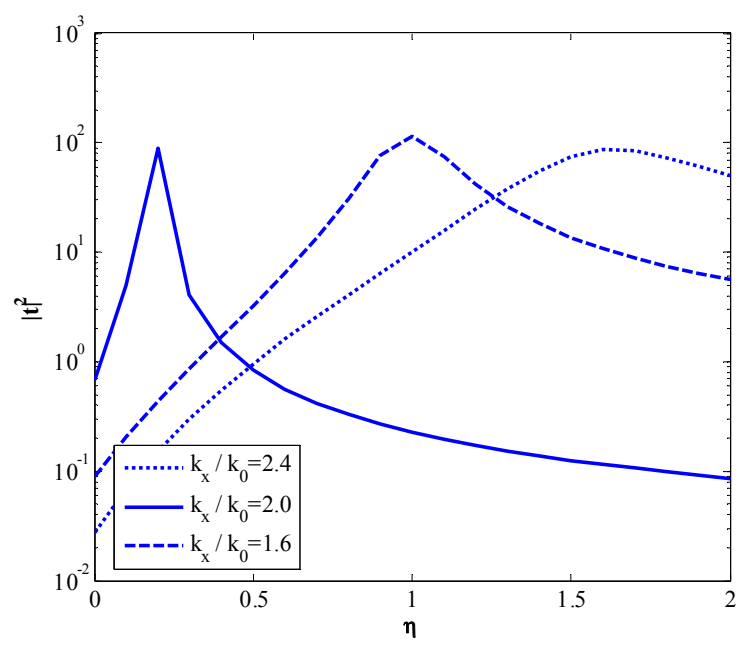

Figure 3. The transmission coefficient for different layer thickness ratios and spatial frequency, for a superlens composed of alternating layers of silver and silica.

In Fig. 2, we show the real part of the in-plane and perpendicular components of the permittivity for different 
layer thickness ratios. From Fig. 2, we can obtain the spatial frequency regions which the permittivity take opposite signs. Fig. 3 shows the transmission coefficient as a function of layer thickness ratios $\eta$ and the spatial frequency for a system composed of alternating layers of silver and silica. As is shown in this figure, for a given spatial frequency $k_{x}$ and a given layer thickness ratio $\eta$, we have the maximum transmission coefficient. In the case of $k_{x}=2.4 k_{0}$, the maximum transmission coefficient is obtained at $\eta=1.6$; In the case of $k_{x}=2.0 k_{0}$, the maximum transmission coefficient is obtained at $\eta=1$; In the case of $k_{x}=1.6 k_{0}$, the maximum transmission coefficient is obtained at $\eta=0.2$. From this figure, we can obtain the optimum layer thickness ratio for a finite total thickness of the superlens at different spatial frequency.

\section{B. Total Thickness of the Multilayered superlenses}

In [5], it is shown that, as the layers are made thinner, the effective-medium approximation becomes more appropriate. For simplicity, we assume that the thickness of each metal layer is maintained at $10 \mathrm{~nm}\left(d_{1}=10 \mathrm{~nm}\right)$, and the thickness of each dielectric layer can be obtained by (3). Therefore, we can increasing or decreasing the total thickness by changing the layer numbers and the layer thickness ratios. In this section, we investigate the effect on imaging performance of varying the total thickness of the superlenses.

Fig. 4 shows the transmission coeffcient intensity versus total silver thicknesses for multilayer superlenses at different working wavelength. We can obtain the total thickness at different working wavelength for maximum transmission. In the case of $325 \mathrm{~nm}$, the transmission coefficient is maximized at $50 \mathrm{~nm}$ total thickness. In the case of $385 \mathrm{~nm}$, the transmission coefficient is maximized at $60 \mathrm{~nm}$ total thickness. If the layer thickness ratio $\eta=1$, that means the layer number of this two cases is 5 and 6 respectively.

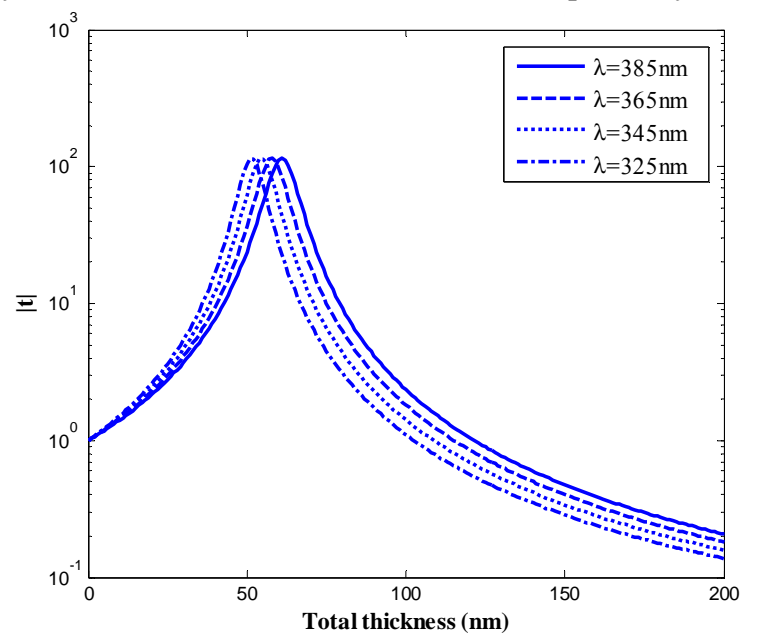

Figure 4. Transmission coe cients as a function of total thicknesses for various working wavelength.

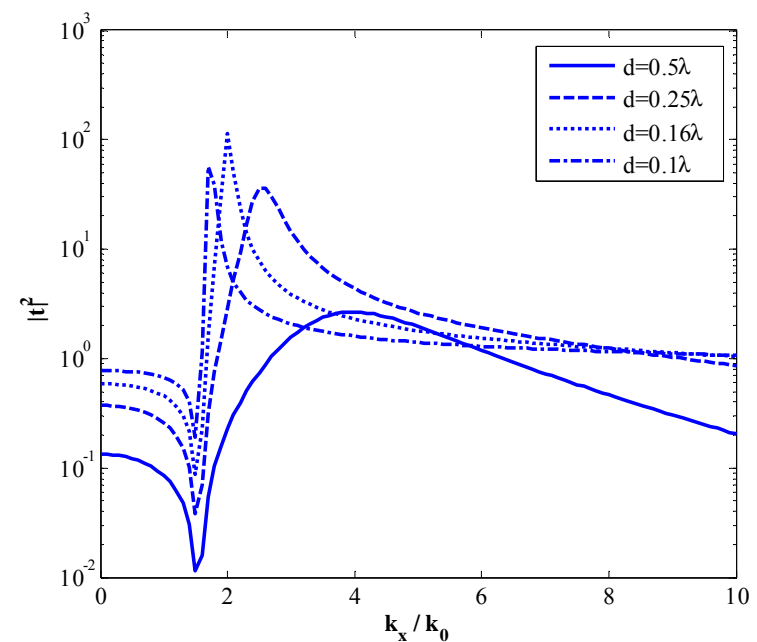

Figure 5. The transmission coefficient as a function of spatial frequency for different total layers thickness, each layer having a thickness of $10 \mathrm{~nm}$.

We then examine the transmission coefficient for different total thickness. Fig. 5 shows the spatial frequency transfer functions for four different superlenses, each of which contains a single layer thickness of $10 \mathrm{~nm}$. As is shown in this figure, each of the superlenses has a significant enhancement in transmission for the evanescent components. In the case of $d=0.16 \lambda$, the peak enhancement result in transmitted intensities is better compared to the other case. However, in the spatial frequency ratio $k_{x} / k_{0}$ range $2.5-8$, the case of $d=0.25 \lambda$ show higher transmission than the others.

\section{Dielectric Materials}

In contrast to pure metal slabs, metal-dielectric composite films are characterized by an effective permittivity $\varepsilon_{e}$ that depends critically on the permittivities and the filling factors of both the metal and dielectric components. We have investigated the effects on imaging performance of changing the layer thickness ratios, which corresponding to the filling factors of the metal and dielectric. In this section, we investigate the imaging performance of a slab with different dielectric materials. We present here the permittivities and layer thickness of three dielectric materials in Table I [8].

TABLE I. PARAMETERS TABLE FOR DIFFERENT DIELECTRIC MATERIALS.

\begin{tabular}{ccc}
\hline \hline Dielectric & Permittivity $\left(\mathcal{E}_{d}\right)$ & Thickness(nm) \\
\hline PMMA & 2.4 & 10 \\
$\mathrm{SiO}_{2}$ & 2.56 & 10 \\
$\mathrm{Al}_{2} \mathrm{O}_{3}$ & 3.217 & 10 \\
\hline \hline
\end{tabular}

The spatial-frequency transfer functions for the three kinds of stacks with different dielectric materials present in Table I are shown in Fig. 6. All superlenses show peak 
enhancements at around $k_{x} / k_{0}=1.8$. From Fig. 6, we can see that the $\mathrm{SiO}_{2}-\mathrm{Ag}$ multilayered superlens is a better choice for a particular transverse wavevector $k_{x}$, but the $\mathrm{Al}_{2} \mathrm{O}_{3}-\mathrm{Ag}$ multilayered superlens provide better enhancement in a wide range spatial frequencies.

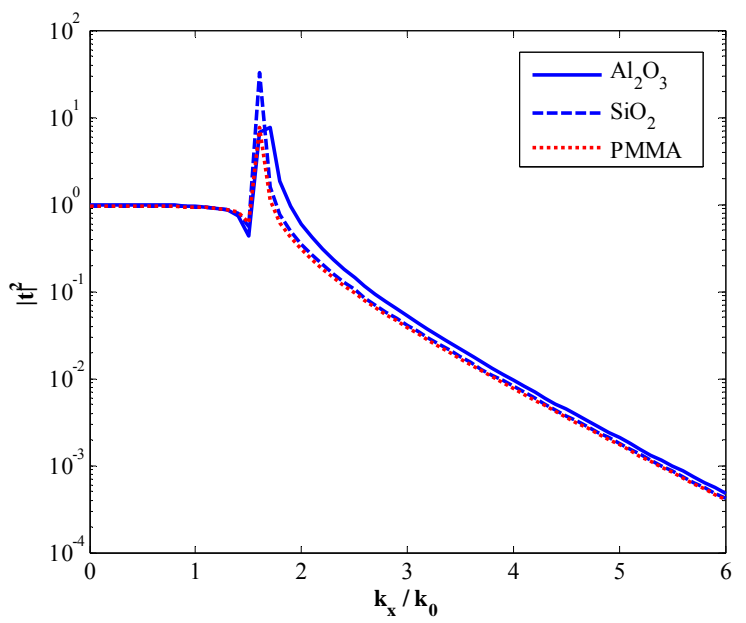

Figure 6. Comparison of the transmission coefficients in $\mathrm{Al}_{2} \mathrm{O}_{3}-\mathrm{Ag} / \mathrm{SiO}_{2}$ Ag/PMMA-Ag multilayered superlenses with total thickness of $80 \mathrm{~nm}$.

\section{RESULT AND DISCUSSION}

We have investigated the effects on imaging performance of varying the structures within a multilayer silver-dielectric superlens. Various structures for changing the layer thickness ratio, the total stack thickness and the dielectric materials have been analyzed in section III. To summarize, for any particular superlens it is possible to optimize the layer thickness ratios, the total stack thickness and the dielectric materials for a optimum imaging performance. We present here an example of a optimized structure based on the above analysis using the FDTD method. We assume a thickness of $60 \mathrm{~nm}$ and layer thickness ratio of 1.2 for multilayer $\mathrm{SiO}_{2}-\mathrm{Ag}$ superlens at the working wavelength of $365 \mathrm{~nm}$. The simulation result is shown in Fig. 7, from which, we can see that it has sharp image of the double slits and high transmittance.

\section{CONCLUSION}

According to the analysis of the imaging performance of various multilayer metallo-dielectric superlenses for nearfield photolithographic imaging, we summarize a optimum structure with particular layer thickness ratio, total stack thickness and dielectric material for a optimum imaging performance. The spatial-frequency transfer functions of these superlenses has strong implications for their utility in practical imaging systems. The FDTD simulation for an optimum example shows sharp image and high tranmittance. Therefore, the analysis results in this paper is helpful for optimization of the structures of multilayer superlenses.

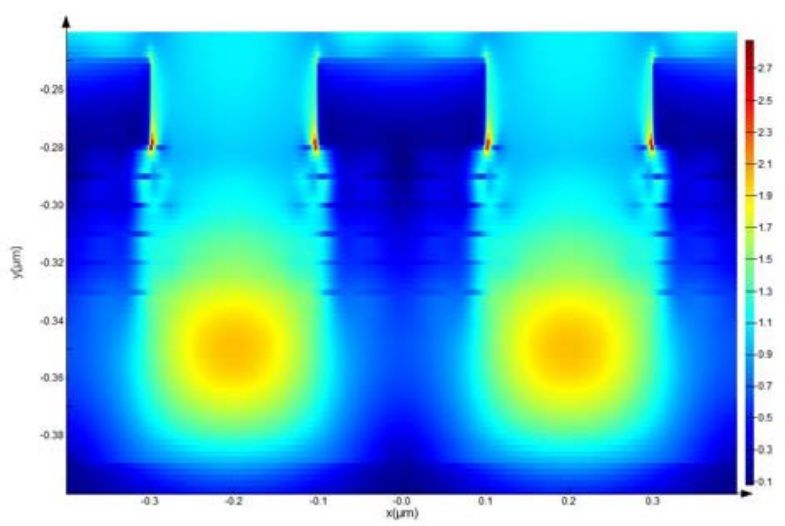

Figure 7. Simulation results of near field imaging in $\mathrm{SiO}_{2}-\mathrm{Ag}$ multilayered superlens devices.

\section{ACKNOWLEDGMENT}

This work was supported by 973 Program of China (No.2011CB301805). The corresponding author is Junxian Ma with advanced technology research centre, Shenzhen University, Shenzhen, China (majx@szu.edu.cn).

\section{REFERENCES}

[1] J. B. Pendry, "Negative refraction makes a perfect lens," Phys. Rev. Lett., vol. 85, pp. 3966-3969, 2000.

[2] S. A. Ramakrishna, J. B. Pendry, W. C. K. Whiltshire and W. Stewart, "Imaging the near field," J. Mod. Optics., vol. 50, pp. 1419-1430, 2003.

[3] R. J. Blaikie and S. J. McNab, "Simulation study of 'perfect lenses' for near-field optical nanolithography," Microelectron. Eng., 6162:97-103, 2002.

[4] D. O. S. Melville and R. J. Blaikie, "Near-field optical lithography using a planar silver lens," J. Vac. Sci. Technol. B, 22(6):3470-3474, 2004.

[5] D. O. S. Melville and R. J. Blaikie, "Analysis and optimization of multilayersilversuperlenses for near-field optical lithography," Physica B 394 (2007) 197-202.

[6] B. Wood ,J. B. Pendry and D. P. Tsai, "Directed subwavelength imaging using a layered metal-dielectric system," Physical Review B 74, $115116(2006) 1-7$

[7] Giaran P. Moore, Matthew D. Arnold, Philip J. Bones and Richard J. Blaikie, "Image fidelity for single-layer and multi-layer silver superlenses," J. Opt. Soc. Am. A, Vol. 25, No. 4, April 2008, 911-918.

[8] Wenshan Cai,Dentcho A. Genov and Vladimir M. Shalaev, "Superlens based on metal-dielectric composites," PHYSICAL REVIEW B 72, 193101 (2005), 1-4.

[9] Weijie Kong, Xiaoping Zhang, Pengfei Cao, Lin Cheng, Qunfeng Shao, Xining Zhao, Li Gong and Xin Jin, "Subwavelength imaging of a multilayered superlens with layers of nonequal thickness," APPLIED OPTICS, Vol. 50, No. 31, November 1st, 2011, 131-136.

[10] Changtao Wang, Yanhui Zhao, Dachun Gan, Chunlei Du, and Xiangang Luo, "Subwavelength imaging with anisotropic structure comprising alternately layered metal and dielectric films," OPTICS EXPRESS, Vol. 16, No. 6, March 17 ${ }^{\text {th }}, 2008,4217-4227$. 